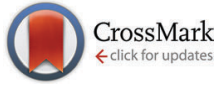

Cite this: Phys. Chem. Chem. Phys., 2015, 17, 2494

Received 10th October 2014, Accepted 25th November 2014

DOI: $10.1039 / c 4 c p 04584 j$

www.rsc.org/pccp

\section{Mass analyzed threshold ionization detected infrared spectroscopy: isomerization activity of the phenol-Ar cluster near the ionization threshold $\dagger$}

\author{
Mitsuhiko Miyazaki, ${ }^{a}$ Shunpei Yoshikawa, ${ }^{a}$ François Michels, ${ }^{b}$ Kentaro Misawa, ${ }^{a}$ \\ Shun-ichi Ishiuchi, ${ }^{a}$ Makoto Sakai, ${ }^{a}$ Otto Dopfer, ${ }^{* c}$ Klaus Müller-Dethlefs ${ }^{* b}$ and \\ Masaaki Fujii*a
}

\begin{abstract}
The structure of the phenol-argon cluster ( $\mathrm{PhOH}-\mathrm{Ar}$ ) in high- $n$ Rydberg states is investigated by the newly developed technique of mass analyzed threshold ionization detected infrared (MATI-IR) spectroscopy. This method selectively measures IR spectra of molecular clusters in very high- $n$ Rydberg states ( $n>100$ ) utilized in zero kinetic energy (ZEKE) photoelectron and MATI spectroscopy, whose ionic cores are essentially the same as the corresponding bare cation. The MATI-IR spectrum exhibits only the free $\mathrm{OH}$ stretching vibration $\left(\nu_{\mathrm{OH}}^{\pi}\right)$ when the $\pi$-bound cluster of the neutral ground electronic state $\left(\mathrm{S}_{0}\right)$ is resonantly excited via the $\mathrm{S}_{1}$ origin to Rydberg states converging to its adiabatic ionization energy level, $\mathrm{IE}_{0}(\pi)$. When Rydberg states converging to vibrationally excited levels of the local $\pi$-bound minimum are prepared, in addition to $\nu_{\mathrm{OH}}^{\pi}$ also the hydrogen-bonded $\mathrm{OH}$ stretching vibration $\left(\nu_{\mathrm{OH}}^{\mathrm{H}}\right)$ of the $\mathrm{H}$-bonded global minimum is observed in the MATI-IR spectra, even for vibrational excitation of only $14 \mathrm{~cm}^{-1}$ above $\mathrm{IE}_{0}(\pi)$. These results show that the $\pi \rightarrow \mathrm{H}$ site switching reaction of the Ar ligand from the aromatic ring to the $\mathrm{OH}$ group proceeds only from vibrationally excited states in the $\pi$-bound cation core with a small barrier of less than $14 \mathrm{~cm}^{-1}$ from $\mathrm{IE}_{0}(\pi)$. On the other hand, directly photoionized $\mathrm{PhOH}^{+}-\mathrm{Ar}$ shows both $\nu_{\mathrm{OH}}^{\mathrm{H}}$ and $\nu_{\mathrm{OH}}^{\pi}$ in the IR spectra, even when it is just ionized to IE $\mathrm{E}_{0}(\pi)$. This result implies that the ionizationinduced $\pi \rightarrow \mathrm{H}$ site switching occurs without excess energy in the $\mathrm{H}$-bound or $\pi$-bound cations, in contrast to very high- $n$ Rydberg states converging to levels of the $\pi$-bound cation. The different efficiencies of the site switching for the Rydberg ion core and the bare ion and the mechanism for the $\pi \rightarrow H$ site switching are interpreted by direct ionization from the $\pi$-bound to the $\mathrm{H}$-bound structures in addition to the conventional vertical ionization and transitions to high- $n$ Rydberg states.
\end{abstract}

\section{Introduction}

Phenol-rare gas clusters $\left(\mathrm{PhOH}-\mathrm{Rg}_{n}\right.$, e.g. $\left.\mathrm{Rg}=\mathrm{Ar}\right)$ are some of the simplest and most popular model systems for studying intermolecular interactions and their competition, because $\mathrm{PhOH}$ has two major intermolecular interaction sites, namely the aromatic $\pi$-cloud and the acidic $\mathrm{OH}$ functional group. The former

\footnotetext{
${ }^{a}$ Chemical Resources Laboratory, Tokyo Institute of Technology,

Yokohama 226-8503, Japan. E-mail: mfujii@res.titech.ac.jp;

Fax: +81-45-924-5250; Tel: +81-45-924-5250

${ }^{b}$ The Photon Science Institute and School of Chemistry,

The University of Manchester, Manchester, M13 9PL, UK.

E-mail: K.Muller-dethlefs@manchester.ac.uk

${ }^{c}$ Institut für Optik und Atomare Physik, Technische Universität Berlin, 10623 Berlin, Germany. E-mail: dopfer@physik.tu-berlin.de

$\dagger$ Electronic supplementary information (ESI) available. See DOI: 10.1039/ c4cp04584j
}

induces strong dispersion interactions, while the latter exhibits mainly hydrogen bonding. The stable structures in various electronic states have extensively been investigated by various spectroscopic techniques to extract fundamental information on the interaction potentials, such as resonance-enhanced multiphoton ionization (REMPI) spectroscopy of the $\mathrm{S}_{1}-\mathrm{S}_{0}$ transition, ${ }^{1,2}$ photoionization efficiency (PIE) measurements, ${ }^{1}$ and infrared (IR) spectroscopy on the $\mathrm{OH}$ stretching vibration $\left(\nu_{\mathrm{OH}}\right) .^{3}$

For the neutral states $\left(S_{0}\right.$ and $\left.S_{1}\right)$, these studies have agreed with the $\pi$-bound $\mathrm{PhOH}-\mathrm{Rg}_{n}$ structures in which the $\mathrm{Rg}$ atoms stick on either sides of the $\pi$-cloud of the phenyl ring. Especially for the smaller Ar clusters with $n=1$ and 2, rotationally resolved UV spectra have provided detailed intermolecular structural parameters. ${ }^{4}$ In the cationic ground states $\left(\mathrm{D}_{0}\right)$, additional induction effects of the positive charge make the $\mathrm{OH}$ group the most stable interaction site. This interaction switching was derived from IR spectra of ionized clusters, $\mathrm{PhOH}^{+}-\mathrm{Rg}$, generated by aggregation 
of $\mathrm{Rg}$ atoms following ionization of $\mathrm{PhOH}$, which leads predominantly to the formation of the most stable isomer. ${ }^{5-13}$ The IR spectra show broad red-shifted hydrogen-bonded $\mathrm{OH}$ stretching vibrations $\left(\nu_{\mathrm{OH}}^{\mathrm{H}}\right)$ of structures in which the $\mathrm{Rg}$ atoms bind to the $\mathrm{OH}$ group. Recent high level $a b$ initio calculations have also supported this switching in the interaction motif upon ionization. $^{14-17}$

On the other hand, photoionization-based spectroscopies of neutral $\pi$-bound $\mathrm{PhOH}-\mathrm{Rg}$ clusters have provided evidence of a further local minimum above the $\pi$-electron system, in addition to the more stable $\mathrm{OH}$ site. Intermolecular vibrations near the adiabatic ionization energy, $\operatorname{IE}_{0}(\pi)$, observed in zero kinetic energy (ZEKE) photoelectron and mass analyzed threshold ionization (MATI) spectroscopies have been well analyzed assuming nearly harmonic progressions of the $\pi$-bound $\mathrm{PhOH}^{+}-\mathrm{Rg}$ structure formed by Franck-Condon restricted photoionization. $^{18-22}$ The first IR spectra of photoionized $\mathrm{PhOH}^{+}-\mathrm{Rg}$ dimers show only the free $\mathrm{OH}$ stretching vibration $\left(\nu_{\mathrm{OH}}^{\pi}\right)$, and no resonances of the H-bound global minima have been identified. ${ }^{3}$ These observations imply that a potential barrier separates the $\pi$-bound and H-bound interaction sites to stabilize the metastable $\pi$-bound structure. The barrier height has been considered to be $>100 \mathrm{~cm}^{-1}$ according to the energy region where discrete intermolecular vibrations are observed in the ZEKE and MATI spectra. ${ }^{18-22}$ This barrier height is consistent with theoretical predictions of the potential curve along the $\pi \rightarrow \mathrm{H}$ isomerization coordinate. ${ }^{14-17}$

Detailed investigation of the IR spectra of $\mathrm{PhOH}^{+}-\mathrm{Rg}$ cation dimers has shown that both the $\pi$-bound and the $\mathrm{H}$-bound structures co-exist even when the $\pi$-bound neutral clusters are ionized with very low ionization excess energy $\left(<5 \mathrm{~cm}^{-1}\right)$ above $\operatorname{IE}_{0}(\pi)$, which is below the lowest vibrational level of $\pi$-bound $\mathrm{PhOH}^{+}-\mathrm{Rg}$ and should be low enough to trap the $\mathrm{Rg}$ atom in the $\pi$-bound local minimum considering the predicted barrier height. 9,11,23 Furthermore, picosecond time-resolved IR spectroscopy has demonstrated that the $\nu_{\mathrm{OH}}^{\mathrm{H}}$ vibration of photoionized $\mathrm{PhOH}^{+}-\mathrm{Kr}$ appears with a time constant of $\sim 20 \mathrm{ps}$ after the photoionization of the neutral $\pi$-bound complex, while the $\nu_{\mathrm{OH}}^{\pi}$ transition is observed immediately after the photoionization event. $^{24}$ The H-bound structure was thought to be produced from the initially prepared $\pi$-bound structure in a $\pi \rightarrow \mathrm{H}$ isomerization reaction and finally the $\mathrm{H} \rightarrow \pi$ back reaction produces an equilibrium population between both isomers. The interpretation of barrierless $\pi \rightarrow \mathrm{H}$ isomerization derived from the IR spectra is in apparent conflict with the interpretation derived from the ZEKE and MATI spectroscopies and also with theoretical studies that predict a barrier height larger than $100 \mathrm{~cm}^{-1} \cdot{ }^{14,15,18-22}$

The analysis of the ZEKE and MATI spectra strongly suggests that there is no dynamics between the $\pi$ - and $\mathrm{H}$-bound minima. In contrast, the time-resolved IR spectra clearly illustrate the $\pi \rightarrow \mathrm{H}$ isomerization. The observed isomerization means that the potential energy surface around the initially prepared $\pi$-bound minimum cannot be described by a simple harmonic potential. Therefore, the spectral analysis of the ZEKE/MATI spectra including the harmonic progressions of the intermolecular vibrations is inconsistent with the IR spectra. Our group has already addressed this contradiction in the structure determination of $\mathrm{PhOH}^{+}-\mathrm{Rg}$ dimers by investigating Rydberg states using autoionization detected IR (ADIR) spectroscopy modified for the measurement of clusters. ${ }^{23}$ ADIR utilizes vibrational autoionization of a Rydberg electron orbiting around the cluster cation core as a messenger to detect the IR absorption, which is probed by the enhancement of the monomer cation signal induced by vibrational predissociation. These ADIR spectra are essentially the same spectra as those of the photoionized $\mathrm{PhOH}^{+}-\mathrm{Rg}$ dimers. We thus concluded that the same isomerization reaction occurs also in the Rydberg states converging to the $\operatorname{IE}_{0}(\pi)$ level of the $\pi$-bonded cluster. However, the inconsistency between the interpretation of the IR and the ZEKE/MATI spectra concerning the isomerization reactivity from the $\mathrm{IE}_{0}(\pi)$ level has not been solved yet.

One problem of the ADIR study stems from its ion detection scheme. ${ }^{23}$ ADIR collects ions produced by both vibrational autoionization and by ionization induced by a perturbation of the Rydberg states such as stray fields. In the previous ADIR study, ${ }^{23} \mathrm{PhOH}-\mathrm{Rg}$ clusters were excited to relatively low Rydberg states $\left(n=\sim 45, \sim 55 \mathrm{~cm}^{-1}\right.$ below $\left.\operatorname{IE}_{0}(\pi)\right)$ to keep interactions between the Rydberg electron and the cation core sufficiently strong for vibrational autoionization and to avoid the contamination from ions produced by direct photoionization. Thus, if $\mathrm{PhOH}^{+}-\mathrm{Rg}$ ions were produced during the excitation process, there might have been the possibility that the ADIR spectra include contributions from IR absorption of $\mathrm{PhOH}^{+}-\mathrm{Rg}$. To discuss and unravel the reaction mechanism precisely, each contribution has to be measured independently.

To this end, in the present study we have developed a new type of IR spectroscopy for ion cores of high- $n$ Rydberg states, which utilizes high-resolution MATI spectroscopy (MATI-IR spectroscopy). The detection scheme of MATI spectroscopy necessarily separates ions produced by direct ionization from ions originating from high- $n$ Rydberg states converging to a single vibrational level of the cation. The MATI signal selectively provides the IR signature of the Rydberg states in the MATI-IR spectrum. IR spectra of directly photoionized cations are also observed simultaneously by monitoring the response of the corresponding direct ionization signal to the IR excitation (REMPI-IR spectroscopy). Therefore, one can separately determine the reaction mechanism of the high- $n$ Rydberg states and the bare cation based on spectroscopic data obtained under the same experimental conditions.

\section{Experimental}

The excitation scheme for the MATI-IR spectroscopy of $\mathrm{PhOH}-\mathrm{Ar}$ is given in Fig. 1 . The parent $\pi$-bound $\mathrm{PhOH}-\mathrm{Ar}$ clusters generated in a molecular beam are resonantly excited to the $S_{1} 0^{0}$ level by the first UV laser pulse $\left(\mathrm{UV}_{\text {exc }}\right)$ and further excited to high- $n$ Rydberg states $(n>100)$ converging to $\operatorname{IE}_{0}(\pi)$ by the second UV laser pulse $\left(\mathrm{UV}_{\text {ion }}\right)$. An IR laser pulse excites the Rydberg states $50 \mathrm{~ns}$ after the UV excitation and its wavelength is scanned through the region of the $\mathrm{OH}$ stretching vibration $\left(\nu_{\mathrm{OH}}\right)$. When the IR laser 


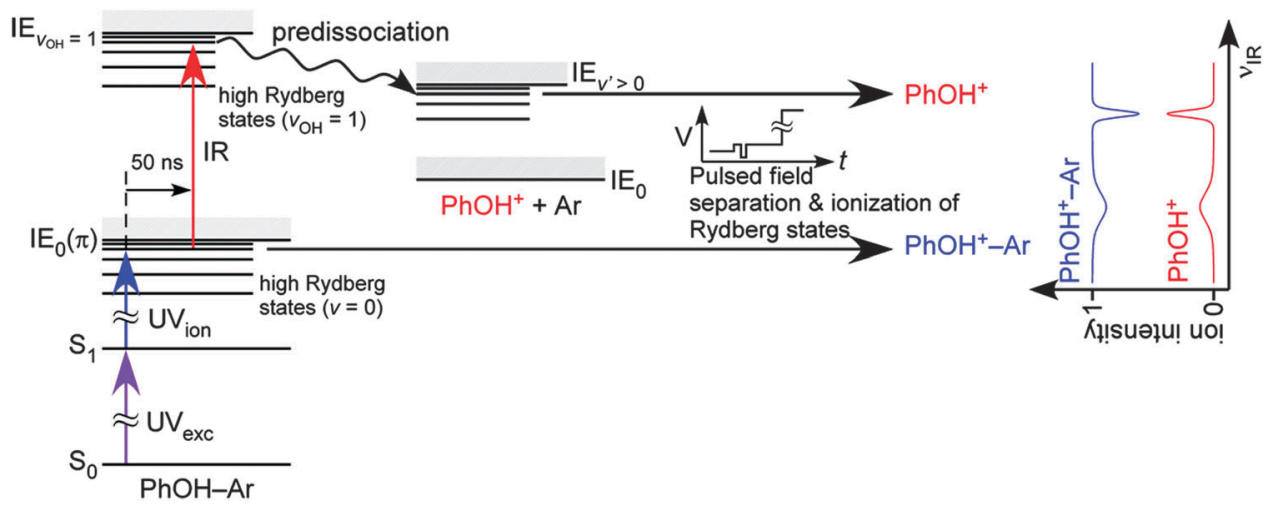

Fig. 1 Excitation scheme for MATI detected IR (MATI-IR) spectroscopy of the PhOH-Ar cluster via the $\mathrm{S}_{1}$ state.

frequency is resonant with $\nu_{\mathrm{OH}}$, vibrational excitation of the ion core occurs and then vibrational predissociation of the Ar atom follows. Since the Rydberg electron in such high- $n$ states has little interaction with the ion core, predissociation results in the production of high- $n$ Rydberg states of bare $\mathrm{PhOH}$.

The Rydberg states are then spatially separated from cations produced directly by the UV ionization process (direct ions) by a MATI separation pulse of $0.383 \mathrm{~V} \mathrm{~cm}^{-1}$ starting $0.5 \mu \mathrm{s}$ after the excitation. To monitor only very high- $n$ Rydberg states (ZEKE states, $n>100$ ) separately from the relatively low- $n$ Rydberg states (MATI states), fractional Stark-state field ionization (FSSFI) is utilized, ${ }^{19}$ a technique which achieves a resolution of MATI spectroscopy comparable to that of ZEKE spectroscopy $\left(<2 \mathrm{~cm}^{-1}\right)$. Shortly, during the separation, only ZEKE states are selectively ionized by applying a weak and short negative pulsed electric field of $-0.667 \mathrm{~V} \mathrm{~cm}^{-1}$ with $60 \mathrm{~ns}$ width that rises at $5.5 \mu$ s after the excitation. Ions generated by this negative pulse are separated by the MATI separation pulse from those MATI states which are still alive. $20 \mu$ s after the excitation, all the components, namely the direct ions and the ions from the ZEKE and the MATI states, are extracted into a reflectron timeof-flight (TOF) mass spectrometer by a strong pulsed electric field $\left(207 \mathrm{~V} \mathrm{~cm}^{-1}\right)$. These three components slightly split a single mass peak of the TOF spectrum into three peaks (see Fig. S1 in the ESI $\dagger$ ). These three peaks provide PIE, highresolution, and normal-resolution MATI spectra, respectively. Thus, by monitoring each TOF peak, the IR absorption of the directly photoionized $\mathrm{PhOH}^{+}-\mathrm{Ar}$ ions, the cationic cores in the ZEKE states, and the cationic cores of the MATI states of $\mathrm{PhOH}-\mathrm{Ar}$ are obtained by depletion of the $\mathrm{PhOH}^{+}-\mathrm{Ar}$ parent and enhancement of the $\mathrm{PhOH}^{+}$fragment mass channels, respectively. The first of them gives the IR spectrum of directly photoionized $\mathrm{PhOH}^{+}-\mathrm{Ar}$, similar to conventional IR photodissociation (REMPI-IR) spectroscopy. ${ }^{3,9,25}$ The second is hereafter called the MATI-IR spectrum. The third component gives the IR signature of MATI states. When we need to distinguish the second and the third component, the former is denoted a high-resolution (HR)-MATI-IR spectrum and the latter a normal-resolution (NR)-MATI-IR spectrum. The simultaneous measurement of MATI-IR and REMPI-IR spectra is a great advantage of this method and enables us to directly compare the dynamics of the ion cores and the directly photoionized ions. The same procedure is applicable for Rydberg states converging to vibrational excited levels of the cationic core. IR resonances of the vibrationally excited ion core are obtained in this case, and the effects of the vibrational excitation on the isomerization reaction can be examined.

The experimental setup used for MATI-IR spectroscopy is essentially the same as that employed previously for MATI spectroscopy of $\mathrm{PhOH}-\mathrm{Ar}_{n}$ clusters. ${ }^{19-22,26-28}$ Tunable $\mathrm{UV}_{\text {exc/ion }}$ laser pulses are generated by frequency doubling of outputs of two Nd:YAGpumped dye lasers (Radiant Dyes: Narrow scan, Continuum: Surelite 10). Tunable IR laser radiation is obtained from the idler output of an IR optical parametric oscillator (LaserVision). The $\mathrm{UV}_{\text {exc }}$ and $\mathrm{UV}_{\text {ion }}$ pulses interact simultaneously with the cluster beam in the acceleration region of a reflectron TOF mass spectrometer, while the IR laser is introduced counter-propagating to the UV beams at a delay of $50 \mathrm{~ns}$. This delay is chosen to avoid the time overlap of the laser pulses. Also, this delay is sufficient to complete the isomerization reaction. ${ }^{24}$ The IR beam is focused on the molecular beam by a $\mathrm{CaF}_{2}$ cylindrical lens with $f=300 \mathrm{~mm}$, while the UV beams are not focused. The IR pulse energy is about $10 \mathrm{~mJ}$ per pulse in the $\nu_{\mathrm{OH}}$ region. The amplified ion signal is averaged and stored in a personal computer through a digital oscilloscope controlled by the computer.

$\mathrm{PhOH}-\mathrm{Ar}$ clusters are generated in a supersonic jet by expanding room temperature $\mathrm{PhOH}$ vapor seeded in a 1/10 gas mixture of $\mathrm{Ar} / \mathrm{Ne}$ through a pulsed valve (General valve: Series 9) into the vacuum chamber at a stagnation pressure of about 0.5 bar. Jet expansion is skimmed by a skimmer with $\phi=1.5 \mathrm{~mm}$ diameter, and the resultant molecular beam is introduced into the ionization region of the TOF mass spectrometer. The system is operated at $10 \mathrm{~Hz}$ repetition rate. $\mathrm{PhOH}$ has been purchased from Sigma-Aldrich and is used without further purification.

\section{Results}

\subsection{REMPI, PIE, and MATI spectra}

Fig. 2(a) shows the two-color two-photon $\left(1+1^{\prime}\right)$ REMPI spectrum of $\mathrm{PhOH}-\mathrm{Ar}$ for $\mathrm{UV}_{\text {ion }}$ set to $32250 \mathrm{~cm}^{-1}$. The $\mathrm{S}_{1}-\mathrm{S}_{0}$ origin band observed at $36315 \mathrm{~cm}^{-1}$ is accompanied by 
transitions assigned to intermolecular modes., ${ }^{1,2,4}$ The clear and background-free appearance of the origin band ensures the clean selection of the $1: 1$ cluster without any fragmentation from higher $1: n$ clusters under the employed experimental conditions. This result is also confirmed by the TOF mass spectrum recorded under the same conditions by setting $\mathrm{UV}_{\text {exc }}$ resonant to the $\mathrm{S}_{1}$ origin, which does not exhibit any $\mathrm{PhOH}-\mathrm{Ar}_{n}$ with $n>1$ (see Fig. S2 in the ESI†). Fig. 2(b) and (c) show the PIE and MATI spectra of $\mathrm{PhOH}-\mathrm{Ar}$ recorded via the $\mathrm{S}_{1}$ origin, respectively. The adiabatic ionization energy, $\operatorname{IE}_{0}(\pi)=$ $68447 \mathrm{~cm}^{-1}$, appears as a sharp step and a peak in the PIE and MATI spectra, respectively. This value matches well with the reported $\mathrm{IE}_{0}(\pi)$ of $\mathrm{PhOH}-\mathrm{Ar}$ when taking into account a field shift of $5 \mathrm{~cm}^{-1}$ in the MATI spectrum. ${ }^{18,22}$ Also the transitions above $\operatorname{IE}_{0}(\pi)$ reproduce the previous reports, ${ }^{19,21,22}$ however with significantly improved spectral quality. These peaks have been assigned to intermolecular vibrations assuming a $\pi$-bound structure in the $\mathrm{D}_{0}$ state, as indicated in Fig. 2(c). ${ }^{18,21}$ The MATI-IR spectra discussed below (Fig. 3) are recorded by setting the frequency of $\mathrm{UV}_{\text {ion }}$ resonant to these peaks with prior excitation to the $S_{1}$ origin.

\subsection{MATI-IR spectra}

The upper trace in Fig. 3(a) shows the MATI-IR spectrum of $\mathrm{PhOH}-\mathrm{Ar}$ obtained by probing the MATI signal at the $\operatorname{IE}_{0}(\pi)$

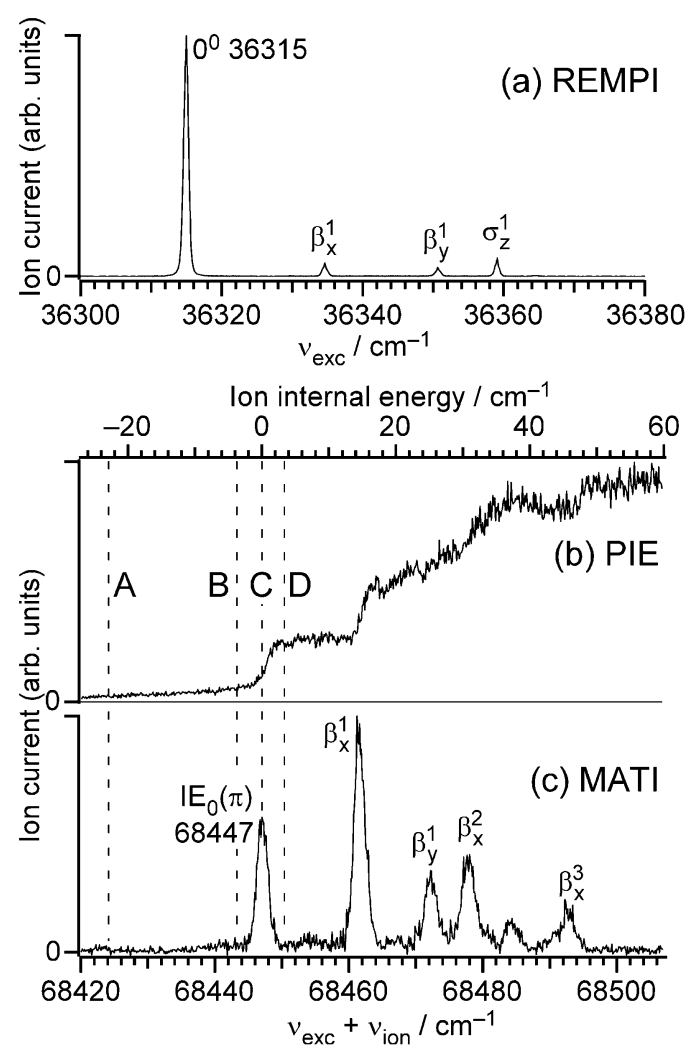

Fig. $21+1^{\prime}$ REMPI spectrum (a), and the PIE (b) and MATI spectra (c) of $\mathrm{PhOH}-\mathrm{Ar}$ recorded via the $\mathrm{S}_{1}$ origin. Dashed lines labeled by $\mathrm{A}-\mathrm{D}$ indicate the ionization positions used for REMPI-IR spectra shown in Fig. 4. The assignments of the intermolecular transitions taken from the literature are indicated. (a) $\mathrm{IE}_{0}(\pi)$
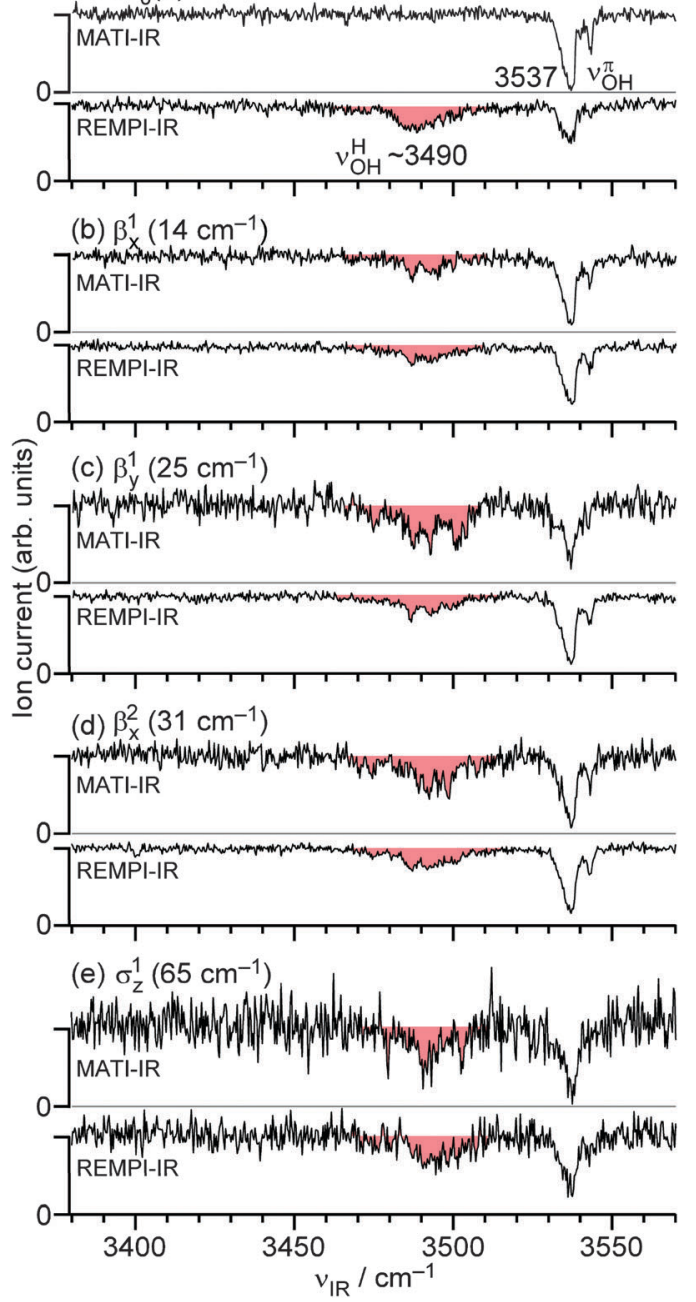

Fig. 3 MATI-IR and REMPI-IR spectra of $\mathrm{PhOH}^{(+)}-\mathrm{Ar}$ obtained by monitoring the parent mass channel at the (a) $I E_{0}(\pi)$, (b) $\beta_{x^{\prime}}^{1}$ (c) $\beta_{y}^{1}$ (d) $\beta_{x}^{2}$ and (e) $\sigma_{z}^{1}$ levels, respectively. The vibrational energies of the levels are given in parentheses.

level. A strong and sharp depletion is observed at $3537 \mathrm{~cm}^{-1}$. This frequency agrees well with $\nu_{\mathrm{OH}}$ of bare $\mathrm{PhOH}^{+}$obtained by ADIR spectroscopy ${ }^{29,30}$ and can be assigned to $\nu_{\mathrm{OH}}^{\pi}$ of the $\pi$-bound $\mathrm{PhOH}^{+}-\mathrm{Ar}$ ion core. The width of the $\nu_{\mathrm{OH}}^{\pi}$ band $\left(\sim 5 \mathrm{~cm}^{-1}\right)$ is, however, smaller than that of the $\nu_{\mathrm{OH}}$ band obtained from ADIR spectroscopy $\left(\sim 20 \mathrm{~cm}^{-1}\right)^{29,30}$ even though the depletion is mostly saturated. This difference has been attributed to electron detachment dynamics in ADIR spectroscopy. ${ }^{29,30}$ The smaller absorption band to the blue side of $\nu_{\mathrm{OH}}^{\pi}$ arises from anharmonic coupling with a so far unassigned vibration. No other absorptions are detected in the lower frequency region down to $3400 \mathrm{~cm}^{-1}$, even though the high power of the IR laser pulse was sufficient to nearly completely saturate the observed $\nu_{\mathrm{OH}}^{\pi}$ band. The same IR spectrum is also obtained as enhancement signal by monitoring the $\mathrm{PhOH}^{+}$fragment channel, which arises from vibrational predissociation (Fig. S4 in the ESI $\dagger$ ). The observation of the single $\nu_{\mathrm{OH}}^{\pi}$ band and the absence of any $\nu_{\mathrm{OH}}^{\mathrm{H}}$ transition means that only the $\pi$-bound structure exists in the ion core of the high- $n$ Rydberg 
states converging to the $\operatorname{IE}_{0}(\pi)$ level of $\mathrm{PhOH}-\mathrm{Ar}$ and that the $\pi \rightarrow \mathrm{H}$ isomerization does not proceed from the $\mathrm{IE}_{0}(\pi)$ level. This result differs from the ADIR spectrum, ${ }^{23}$ in which the contributions are detected as $\nu_{\mathrm{OH}}^{\pi}$ and $\nu_{\mathrm{OH}}^{\mathrm{H}}$, although both methods monitor the IR absorption of the cation core of Rydberg states converging to the $\mathrm{IE}_{0}(\pi)$ level. We discuss this inconsistency below.

Vibrational excitation qualitatively changes the appearance of the IR spectra even for very small energies. The upper traces in Fig. 3(b)-(e) show MATI-IR spectra obtained by exciting the intermolecular vibrational levels $\beta_{x}^{1}\left(14 \mathrm{~cm}^{-1}\right), \beta_{y}^{1}\left(25 \mathrm{~cm}^{-1}\right), \beta_{x}^{2}$ $\left(31 \mathrm{~cm}^{-1}\right)$, and $\sigma_{z}^{1}\left(65 \mathrm{~cm}^{-1}\right)$ in the cationic state, respectively. The $\sigma_{z}^{1}$ Rydberg states are realized through excitation via the very weak $S_{1} \sigma_{z}^{1}$ band, and the corresponding MATI spectrum is available in Fig. S3 in the ESI. $\dagger$ In striking contrast to the MATI-IR spectrum of $\operatorname{IE}_{0}(\pi)$, the MATI-IR spectra involving intermolecular excitations exhibit red-shifted and broad absorptions at around $3490 \mathrm{~cm}^{-1}$, in addition to $\nu_{\mathrm{OH}}^{\pi}$ at $3537 \mathrm{~cm}^{-1}$ which still remains the strongest feature. The former can be assigned to $\nu_{\mathrm{OH}}^{\mathrm{H}}$, because its frequency matches with $\nu_{\mathrm{OH}}^{\mathrm{H}}$ of ionized $\mathrm{PhOH}^{+}-\mathrm{Ar}^{5-8}$ The observation of $\nu_{\mathrm{OH}}^{\mathrm{H}}$ indicates the occurrence of $\pi \rightarrow \mathrm{H}$ isomerization, since the Rydberg states are prepared by Franck-Condon restricted photoexcitation from the $\pi$-bound $\mathrm{S}_{1}$ state. The $\nu_{\mathrm{OH}}^{\mathrm{H}}$ frequency observed here $\left(\sim 3490 \mathrm{~cm}^{-1}\right)$ is somewhat blue-shifted from that observed for cold $\mathrm{PhOH}^{+}-\mathrm{Ar}$ clusters generated by collisional aggregation of $\mathrm{PhOH}^{+}$and $\mathrm{Ar}\left(\nu_{\mathrm{OH}}^{\mathrm{H}}=3468 \mathrm{~cm}^{-1}\right) \cdot{ }^{5-8}$ This blue-shift has been interpreted as weakening of the $\mathrm{H}$-bond in hot clusters caused by redistribution of internal energy during the course of the exothermic $\pi \rightarrow \mathrm{H}$ isomerization reaction $\left(100-300 \mathrm{~cm}^{-1}\right)^{14,15,17,28}$ among the three intermolecular modes. ${ }^{8,9}$ The lowest-frequency vibration of $\mathrm{PhOH}^{+}$has $\sim 180 \mathrm{~cm}^{-131}$ and is the only intramolecular vibration, which can be excited by the excess energy available in this reaction. The correspondence of the $\nu_{\mathrm{OH}}^{\mathrm{H}}$ frequency of the core excited Rydberg states and directly photoionized $\mathrm{PhOH}^{+}-\mathrm{Ar}^{8,11,23}$ also supports the conclusion that the $\mathrm{H}$-bound structure is produced by the isomerization. These observations imply the existence of a tiny activation barrier of less than $14 \mathrm{~cm}^{-1}$ from $\operatorname{IE}_{0}(\pi)$ for the $\pi \rightarrow \mathrm{H}$ isomerization in the ion core, and that the reaction is promoted by vibrational excess energy which is sufficient to surmount this barrier. The derived barrier height is quite low compared to the theoretical estimation of $\sim 100 \mathrm{~cm}^{-1}$ derived from the CCSD(T)/CBS limit extrapolation. ${ }^{14,15}$ Careful consideration of higher terms in the potential such as zero point vibrational energy, anharmonicity, and coupling between the three intermolecular modes and also the intramolecular vibrations may resolve this slight inconsistency between experiment and current prediction.

The ratio of the integrated band intensity of the $\nu_{\mathrm{OH}}^{\mathrm{H}}$ and $\nu_{\mathrm{OH}}^{\pi}$ transitions tends to increase monotonically with the vibrational energy (except for $\beta_{y}^{1}$ ), and a mode-selective dependence is not obvious (Table 1). The yield of the reaction, however, is far from unity even for excitation above the barrier. The experimental integrated intensity of $\nu_{\mathrm{OH}}^{\mathrm{H}}$ is of the same order as that of $\nu_{\mathrm{OH}}^{\pi}$, although the IR oscillator strength of $\nu_{\mathrm{OH}}^{\mathrm{H}}$ has been estimated to be about two times stronger than that of $\nu_{\mathrm{OH}}^{\pi} \cdot{ }^{5,8}$ From these values, the relative population of the $\mathrm{H}$-bound structure
Table 1 Experimental relative integrated areas of the $\nu_{\mathrm{OH}}^{\mathrm{H}}$ and $\nu_{\mathrm{OH}}^{\pi}$ transitions in the MATI-IR spectra (Fig. 3) ${ }^{a}$

\begin{tabular}{ll}
\hline Vibration level (energy) & $\begin{array}{l}\text { Relative integrated } \\
\text { area }\left(A_{\mathrm{OH}}^{\mathrm{H}} / A_{\mathrm{OH}}^{\pi}\right)\end{array}$ \\
\hline $\mathrm{IE}_{0}(\pi)\left(0 \mathrm{~cm}^{-1}\right)$ & 0.0 \\
$\beta_{x}^{1}\left(14 \mathrm{~cm}^{-1}\right)$ & 0.5 \\
$\beta_{y}^{1}\left(25 \mathrm{~cm}^{-1}\right)$ & 2.0 \\
$\beta_{x}^{2}\left(31 \mathrm{~cm}^{-1}\right)$ & 1.0 \\
$\sigma_{z}^{1}\left(65 \mathrm{~cm}^{-1}\right)$ & 0.9 \\
${ }^{a}$ Integration ranges are $3470-3510 \mathrm{~cm}^{-1}$ and $3530-3550 \mathrm{~cm}^{-1}$ for $\nu_{\mathrm{OH}}^{\mathrm{H}}$ \\
and $\nu_{\mathrm{OH}}^{\pi}$, respectively.
\end{tabular}

generated by the reaction is then obtained as 0.5 of that of the $\pi$-bound population. This limited reaction yield has been interpreted by a reaction model including $\pi \rightarrow \mathrm{H}$ forward and $\mathrm{H} \rightarrow \pi$ backward reactions, and the lack of intracluster vibrational energy redistribution (IVR) from the reaction coordinate. $^{8,24,32}$

These results of the Rydberg states reflect exactly the reactivity of the $\mathrm{PhOH}^{+}$-Ar cation. This fact is proven by checking the dependence of the results on the principal quantum number $(n)$. NR-MATI-IR spectra obtained by monitoring the lower- $n$ MATI states (shown in Fig. S4 in the ESI $\dagger$ ) provide the same result as the HR-MATI-IR spectra probing the high- $n$ ZEKE states. This means that the interaction of the Rydberg electron with the ion core has already (nearly) vanished even for the MATI states and the ion core can be considered to be the bare cation, corresponding to the usual intuition of Rydberg states. $^{33}$

\subsection{REMPI-IR spectra}

The lower trace in Fig. 3(a) shows the REMPI-IR spectrum of $\mathrm{PhOH}^{+}-\mathrm{Ar}$ ionized just to the $\mathrm{IE}_{0}(\pi)$ level, recorded simultaneously with the MATI-IR spectrum in Fig. 3(a). Surprisingly, even without any excess energy a broad $\nu_{\mathrm{OH}}^{\mathrm{H}}$ band can clearly be recognized at around $3490 \mathrm{~cm}^{-1}$ in addition to the sharp $\nu_{\mathrm{OH}}^{\pi}$ band at $3537 \mathrm{~cm}^{-1}$. This REMPI-IR spectrum therefore implies $\pi \rightarrow \mathrm{H}$ site-switching without any activation barrier for directly photoionized $\mathrm{PhOH}^{+}-\mathrm{Ar}$. The blue-shifted position of $\nu_{\mathrm{OH}}^{\mathrm{H}}$ rules out the possibility of contamination from higher $\mathrm{PhOH}-\mathrm{Ar}_{n}$ clusters with $n \geq 2$. Evaporation of $\mathrm{Ar}$ atoms removes excess energy of resulting $\mathrm{PhOH}^{+}-\mathrm{Ar}$ fragment ions, and $\nu_{\mathrm{OH}}^{\mathrm{H}}$ appears as a much sharper transition at $\sim 3470 \mathrm{~cm}^{-1}$ similar to the case of clusters formed by Ar aggregation to $\mathrm{PhOH}^{+} .8,25,34$ This result is consistent with the previous REMPI-IR and ADIR studies $^{9,11,23,24}$ but contradicts the MATI-IR data mentioned above for excitation of $\operatorname{IE}_{0}(\pi)$. Moreover, the intensities of both bands are comparable, indicating a higher reaction yield than those observed in the MATI-IR spectra. Vibrational excitation, however, weakens the intensity of $\nu_{\mathrm{OH}}^{\mathrm{H}}$ and enhances $\nu_{\mathrm{OH}}^{\pi}$, and the appearance of the REMPI-IR spectra becomes very similar to that of the corresponding MATI-IR spectra as shown in Fig. 3(b)-(e). This result means that the reaction yield is lowered for these levels compared to the $\operatorname{IE}_{0}(\pi)$ level despite their higher excess energy. This behavior of the REMPI-IR spectra is in marked contrast to that of the MATI-IR spectra, 
with particularly striking differences for excitation at the $\operatorname{IE}_{0}(\pi)$ level, although the ion core in high- $n$ Rydberg states must be identical to the bare cations.

To clarify the origin of the difference in REMPI-IR and MATI-IR spectra, the excess energy dependence of the REMPI-IR spectra is investigated by off-resonating the frequency of $\nu_{\text {ion }}$ around $\operatorname{IE}_{0}(\pi)$. The chosen $\nu_{\text {ion }}$ frequencies are (a) far below $\left(-22.9 \mathrm{~cm}^{-1}\right)$, (b) just below $\left(-3.75 \mathrm{~cm}^{-1}\right)$, (c) directly at, and (d) just above $\left(+3.35 \mathrm{~cm}^{-1}\right)$ the $\operatorname{IE}_{0}(\pi)$ band. These ionization positions are indicated by dashed lines labeled A-D in Fig. 2(b). These measured REMPI-IR spectra are compared in Fig. 4(a)-(d), respectively. Nearly all spectra show both $\nu_{\mathrm{OH}}^{\mathrm{H}}$ and $\nu_{\mathrm{OH}}^{\pi}$, similar to the REMPI-IR spectra shown in Fig. 3. The high $\nu_{\mathrm{OH}}^{\mathrm{H}}$ frequency $\left(3490 \mathrm{~cm}^{-1}\right)$ implies that the probed $\mathrm{H}$-bound structure possesses a large internal energy. A striking feature is that these spectra show a drastic alternation of the relative intensities of $\nu_{\mathrm{OH}}^{\mathrm{H}}$ and $\nu_{\mathrm{OH}}^{\pi}$ across $\operatorname{IE}_{0}(\pi)$. When $\mathrm{PhOH}-\mathrm{Ar}$ is excited to $-22.9 \mathrm{~cm}^{-1}$ below $\operatorname{IE}_{0}(\pi)$, the REMPI-IR spectrum shows a strong $\nu_{\mathrm{OH}}^{\mathrm{H}}$ transition centered at $3490 \mathrm{~cm}^{-1}$ and the $\nu_{\mathrm{OH}}^{\pi}$ band $\left(3537 \mathrm{~cm}^{-1}\right)$ is not visible. The latter gains weak intensity for ionization just below $\operatorname{IE}_{0}(\pi)\left(3.75 \mathrm{~cm}^{-1}\right)$, and is almost as strong as $\nu_{\mathrm{OH}}^{\mathrm{H}}$ in the case of just threshold ionization $\left(0 \mathrm{~cm}^{-1}\right)$. Furthermore, the intensities are suddenly largely reversed when the ionization exceeds the $\operatorname{IE}_{0}(\pi)$ band by only $3.35 \mathrm{~cm}^{-1}$.

The observed intensity change in the REMPI-IR spectra in Fig. 4 means that below $\operatorname{IE}_{0}(\pi)$ the generated isomer population concentrates on the H-bound structures and is inverted for $\operatorname{IE}_{0}(\pi)$. The small population of the $\pi$-bound structure below $\operatorname{IE}_{0}(\pi)$ is reasonable because the energy is not sufficient for the ionization of the $\pi$-bound structure. No clear explanation of the weak appearance of $\nu_{\mathrm{OH}}^{\pi}$ in the case of the ionization at

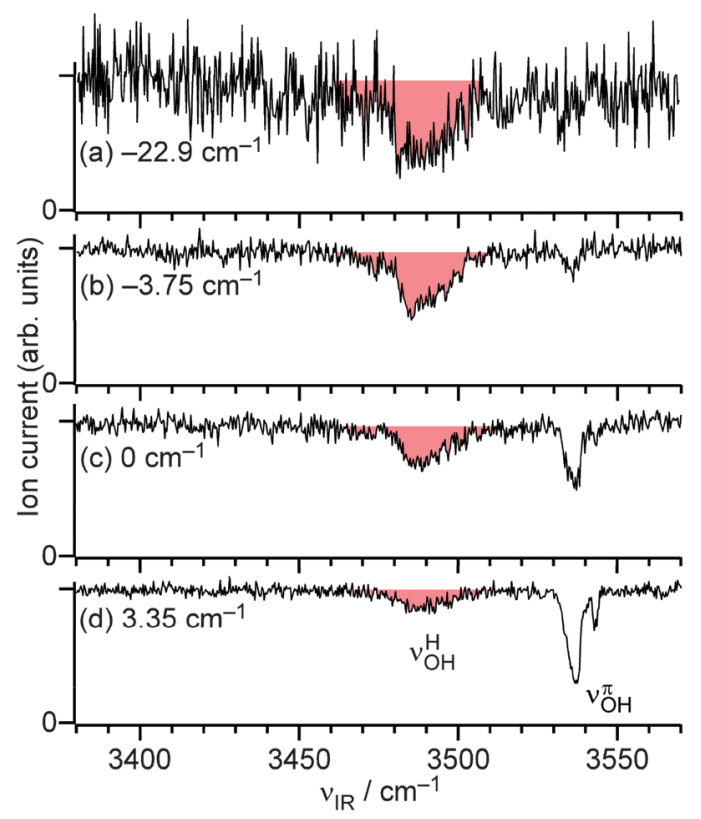

Fig. 4 REMPI-IR spectra of $\mathrm{PhOH}^{+}-\mathrm{Ar}$ obtained by ionizing near the $\mathrm{IE}_{0}(\pi)$ level. The ionization energy relative to the $\mathrm{IE}_{0}(\pi)$ level is indicated for each trace. These positions correspond to the dashed lines A-D in Fig. 2(b).
$-3.75 \mathrm{~cm}^{-1}$ is possible at this moment. One possibility might be a migration of the Ar atom to a position far from the $\mathrm{H}$-bound site. At this energy, Ar can travel from the H-bound site to positions very close to the $\pi$-bound site where the $\mathrm{H}$-bond interaction is negligible. ${ }^{8}$ Such states may have IR signatures at $\nu_{\mathrm{OH}}^{\pi}$, and this signal can be observed in the IR spectrum. A DFT calculation has estimated that an out-of-plane displacement of $\sim 200 \mathrm{pm}$ from the $\mathrm{H}$-bound global minimum almost quenches the red shift of $\nu_{\mathrm{OH}}$, although further displacement of more than $300 \mathrm{pm}$ to the center of the aromatic ring is necessary for reaching the $\pi$-bound local minimum structure. $^{8}$

\section{Discussion}

The appearance of the H-bound isomer below $\operatorname{IE}_{0}(\pi)$ is quite peculiar. The adiabatic ionization energy of the H-bound structure, $\operatorname{IE}_{0}(\mathrm{H})$, is lower than $\operatorname{IE}_{0}(\pi)$. According to the theoretical estimation, the difference is about $100-300 \mathrm{~cm}^{-1} \cdot 14,15,17,28$ Thus, in principle, energetically the H-bound structure can readily be ionized at these ionization positions. On the other hand, the H-bound structure has a largely different geometry than the $\pi$-bound isomer in $S_{1}$ from which the photoionization originates. Therefore, one can assume that the poor FranckCondon overlap between the $\pi$-bound and the H-bound structures essentially forbids the ionization to the adiabatic $\mathrm{H}$-bound threshold. The experimental IR results, however, strongly suggest direct ionization to the $\mathrm{H}$-bound structure from the $\pi$-bound structure. Actually, the PIE spectrum in Fig. 2(b) exhibits indeed a weak and broad signal starting from energies well below $\operatorname{IE}_{0}(\pi)$ (at least more than $20 \mathrm{~cm}^{-1}$ ) and gradually increasing toward higher frequency. PIE curves often show a red tail below the corresponding $\mathrm{IE}_{0}$ due to field ionization of long-lived Rydberg states. In the case of $\mathrm{PhOH}^{+}-\mathrm{Ar}$, the decreasing signal continues however much further down to the red from $\operatorname{IE}_{0}(\pi)$ when compared to the corresponding PIE spectrum of the $\mathrm{PhOH}^{+}$monomer, despite the field-free excitation conditions employed. ${ }^{35}$ Such a broad appearance of a PIE spectrum corresponds well to clusters, in which the structures in the neutral and ionic states are completely different. A good example for such a cluster is the benzene-water dimer, $\mathrm{Bz}-\mathrm{H}_{2} \mathrm{O} .{ }^{36-40}$ In the neutral state the water molecule binds above the aromatic $\pi$ ring forming an $\mathrm{OH}-\pi$ hydrogen bond, while in the cationic state the water is located in the plane of the benzene ring and forms two bifurcated $\mathrm{CH}-\mathrm{O}$ hydrogen bonds. As a consequence, vertical ionization of $\mathrm{Bz}-\mathrm{H}_{2} \mathrm{O}$ brings the cluster to a very high energy part of the interaction potential, because the positively charged hydrogen atoms of the water molecule point toward the positive charge generated by the ionization of benzene. Therefore, almost all the clusters dissociate after the ionization, and only $\sim 10 \%$ of them survive the ionization-induced $\pi \rightarrow \mathrm{CH}$ isomerization. ${ }^{36,37}$ This isomerization path is quite similar to the $\pi \rightarrow \mathrm{H}$ geometry change in $\mathrm{PhOH}-\mathrm{Ar}$ induced by ionization. The PIE spectrum of $\mathrm{Bz}-\mathrm{H}_{2} \mathrm{O}$ shows only a very slow monotonic rise. ${ }^{38}$ Such a slow rise corresponds well to the broad PIE signal observed in the 
present study for $\mathrm{PhOH}-\mathrm{Ar}$, although sharp steps are superimposed from additional ionization to the $\pi$-bound local minimum. $\mathrm{Bz}^{+}-\mathrm{H}_{2} \mathrm{O}$ does not have such a local minimum at the Franck-Condon region. The probability for such a direct ionization step into the $\mathrm{H}$-bound cation structure starting from the neutral $\pi$-bound $\mathrm{S}_{1}$ structure of $\mathrm{PhOH}-\mathrm{Ar}$ is of course very small but nonzero. In this case, ionization occurs mainly to highly excited intermolecular vibrational states of the $\mathrm{H}$-bound $\mathrm{PhOH}^{+}$-Ar cation, whose wavefunctions have a small but nonvanishing overlap with the wavefunction of the vibrationless $S_{1}$ level of $\pi$-bound $\mathrm{PhOH}-\mathrm{Ar}$ with Ar centered above the aromatic ring. Such an overlap occurs in the tail of the $S_{1}$ origin wavefunction, which extends to the region above the oxygen atom of $\mathrm{PhOH}$ near the top of the barrier, ${ }^{14}$ and enables overlap with the wavefunctions of the cationic H-bound structure with high excitation of the intermolecular bending mode, which corresponds closely to the site-switching coordinate. This process leads to vertical ionization from the edge of the $\pi$-bound wavefunction in $S_{1}$ to a point beyond the barrier in the cationic state, because the strong attraction by the $\mathrm{OH}$ group shifts the separation between the H-bound and $\pi$-bound structures in the $\mathrm{D}_{0}$ state much further toward the $\pi$ ring. The REMPI-IR spectra in the present study suggest that this type of direct ionization plays an important role in the site switching.

Another possibility to explain the IR spectra below $\operatorname{IE}_{0}(\pi)$ is autoionization of high- $n$ Rydberg states converging to the vibrational excited levels of the cation. When high- $n$ Rydberg states in the region higher than $\operatorname{IE}_{0}(\mathrm{H})$ are prepared, the ion can be produced by vibrational autoionization. Energetically only the $\mathrm{H}$-bound cation is accessible from the high- $n$ Rydberg states, therefore only $\nu_{\mathrm{OH}}^{\mathrm{H}}$ can be observed in the IR spectra. Although this autoionization mechanism cannot be completely ruled out, the contribution of this process is believed to be small. The autoionization efficiency is proportional to $1 / n^{3} \cdot{ }^{41}$ Furthermore, their oscillator strength is also a function of $1 / n^{3} .{ }^{42}$ Thus the overall ion yield from this mechanism has to drop down rapidly toward $\operatorname{IE}_{0}(\pi)$. In contrast, the observed broad signal below $\operatorname{IE}_{0}(\pi)$ is increasing toward $\operatorname{IE}_{0}(\pi)$. This observation indicates that the autoionization mechanism provides at most a minor contribution.

When including the process for direct ionization to H-bound structures described above, all observed results can qualitatively be explained (Fig. 5). For ionization energies below $\operatorname{IE}_{0}(\pi)$, only the H-bound cation can be produced by direct ionization. In this case, only $\nu_{\mathrm{OH}}^{\mathrm{H}}$ is observed in the REMPI-IR spectrum. Because this process is adiabatic ionization to the H-bound structure, no $\pi \rightarrow \mathrm{H}$ isomerization dynamics can be observed in time resolved experiments. When the ionization energy is between $\operatorname{IE}_{0}(\pi)$ and the $\beta_{x}^{1}$ level, the ionization channel to the zero-point level of the $\pi$-bound cation is open, in addition to the direct ionization channel into the $\mathrm{H}$-bound cation. Isomerization from the $\operatorname{IE}_{0}(\pi)$ level, however, is prevented by the nonvanishing barrier. Tunneling through the barrier can be neglected on the timescale of the experiment, and the wavefunction of the $\operatorname{IE}_{0}(\pi)$ level has negligible amplitude for $\mathrm{H}$-bound geometries. Thus, the MATI-IR spectrum that selectively monitors Rydberg

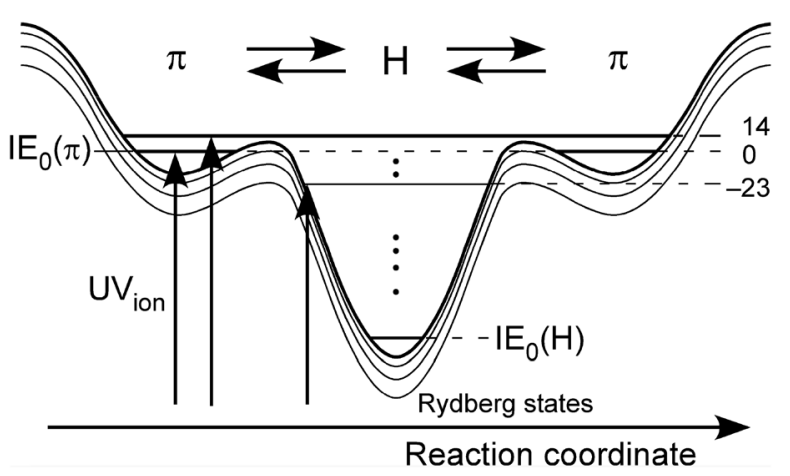

Fig. 5 Schematic potential curve for the $\pi \rightarrow \mathrm{H}$ site switching of $\mathrm{PhOH}^{+}-$ Ar. Values on the right hand side show the energy of the levels relative to $\mathrm{IE}_{0}(\pi)$ in $\mathrm{cm}^{-1}$

states of the $\pi$-bound cation exhibits only the $\nu_{\mathrm{OH}}^{\pi}$ band. The wavefunction of the $\pi$-bound structure above the ring prepared in this way has the same amplitude as the second $\pi$ site below the ring because of the $C_{\mathrm{s}}$ symmetry of the intermolecular potential, and also has a very small amplitude at the H-bound structure. Indeed, the coupling of the states in both equivalent $\pi$ minima gives rise to a tiny tunneling splitting of the $\operatorname{IE}_{0}(\pi)$ level, whose magnitude strongly increases for the intermolecular bending states along the reaction coordinate. The MATI-IR spectrum, however, shows that the lifetime of the populated $\pi$-bound state is longer than $50 \mathrm{~ns}$, which is the delay between $\nu_{\mathrm{exc} / \mathrm{ion}}$ and $\nu_{\mathrm{IR}}$. This observation means that tunneling through the reaction barrier is effectively quenched by the heavy mass of Ar, even for a barrier height of less than $14 \mathrm{~cm}^{-1}$ from $\mathrm{IE}_{0}(\pi)$. The REMPI-IR spectrum on the other hand probes both ionization paths, and hence shows both $\nu_{\mathrm{OH}}^{\mathrm{H}}$ and $\nu_{\mathrm{OH}}^{\pi}$ in the spectrum. This spectral appearance seems to suggest that isomerization occurs, but the $\nu_{\mathrm{OH}}^{\mathrm{H}}$ band results in fact from direct ionization (involving an adiabatic $\pi \rightarrow \mathrm{H}$ site-switch) and the $\nu_{\mathrm{OH}}^{\pi}$ band originates from ionization to the $\pi$-bound structure. Therefore, no dynamics also occurs in these cases. The relative intensities of the two bands in the REMPI-IR spectra simply reflect relative Franck-Condon factors for the processes at the given ionization energy. When the ionization energy exceeds $14 \mathrm{~cm}^{-1}\left(\beta_{x}^{1}\right.$ level), then ionization to levels above the reaction barrier is possible. The wavefunctions of these states cover both the H-bound and $\pi$-bound structures. Thus, the final levels reached by the ionization become common, and the same state is prepared in both ionization processes (MATI and PIE). The similar appearance of MATI-IR and REMPI-IR spectra for these vibrational levels supports this mechanism. In this case, the ionization results in the preparation of a wave packet whose probability density is initially localized on the $\pi$-bound structure reflecting the larger $\pi-\pi$ Franck-Condon overlap from the $S_{1}$ origin level compared to that of the $\pi-H$ overlap. Subsequently, the wave packet propagates toward the H-bound structure and finally spreads out over the $\pi$-bound and H-bound structures on the picosecond timescale. ${ }^{24}$ IR spectra measured using nanosecond lasers observe states after the delocalization of the wavefunction and thus show both the $\nu_{\mathrm{OH}}^{\mathrm{H}}$ and the $\nu_{\mathrm{OH}}^{\pi}$ bands. Picosecond time-resolved IR spectroscopy enables us to trace the 
propagation dynamics in real time, and the previous report on $\mathrm{PhOH}^{+}-\mathrm{Kr}$ is considered to monitor this process at an ionization excess energy of $60 \mathrm{~cm}^{-1} \cdot{ }^{24}$

One further issue to be addresses is a possible contribution from Rydberg states converging to levels of the H-bound cation. If direct ionization of the $\mathrm{H}$-bound structure from the $\pi$-bound structure is possible, then also Rydberg states of the H-bound structure can be generated. Similar to the PIE curve, these states may give rise to a broad spectral feature below $\operatorname{IE}_{0}(\pi)$ to the MATI spectrum. Such a signal, however, has never been observed so far. This difference between the MATI and PIE spectra can be explained by the different mechanisms by which their signal intensities are generated. The relative peak intensities in MATI spectra are determined by Franck-Condon factors between the initial and final levels, while the intensity in the PIE spectrum corresponds to the integral of all these peaks below the employed ionizing photon energy. In the case of direct ionization from the $\pi$-bound to the H-bound structures, the Franck-Condon factors for every single transition are very small and their peak heights in the MATI spectra are too low to be detected and resolved at the available detection sensitivity. On the other hand, the state density is very high because the ionization occurs close to the $\pi \rightarrow \mathrm{H}$ reaction barrier, where intermolecular bending vibrations of the $\mathrm{H}$-bound structure are highly excited with large amplitude motion toward the $\pi$-bound structure. The integral of the Franck-Condon factors over all these many levels can provide sufficient signal intensity to be detected in the PIE spectrum.

This explanation also resolves the inconsistency between the MATI-IR and ADIR spectra mentioned above. ADIR spectroscopy collects ions produced by autoionization and direct ionization without separation. ${ }^{29,30}$ The previous ADIR experiment observed the IR absorption of $\mathrm{PhOH}-\mathrm{Ar}$ ion cores in Rydberg states converging to $\operatorname{IE}_{0}(\pi)$ as an enhanced signal of bare $\mathrm{PhOH}^{+}$, which results from autoionization of Rydberg states generated by vibrational predissociation of the cluster core, achieved by a pulsed extraction field. ${ }^{23}$ At that time, the laser intensities of $\nu_{\text {exc }}$ and $\nu_{\text {ion }}$ were reduced as much as possible so that no signal appears from the UV lasers alone. Under these conditions, $\mathrm{PhOH}$ could not be ionized because $\nu_{\text {exc }}$ was off-resonant and $\nu_{\text {ion }}$ did not reach $\mathrm{IE}_{0}$. For $\mathrm{PhOH}-\mathrm{Ar}$, the extraction pulse was delayed by $250 \mathrm{~ns}$, which is late enough for the prepared Rydberg states to decay $(n \sim 45$, $\sim 55 \mathrm{~cm}^{-1}$ below $\operatorname{IE}_{0}(\pi)$ ), because their lifetime is observed to be shorter than a few nanoseconds from the ADIR experiment. When Rydberg states of only the $\pi$-bound structure are prepared by the excitation, the ADIR spectrum should match with the present MATI-IR spectrum. On the other hand, if $\mathrm{H}$-bound cations are generated through the broad feature of the PIE curve, their IR absorption also produces $\mathrm{PhOH}^{+}$. $\mathrm{PhOH}^{+}$produced in this way is not distinguished from that of autoionization in ADIR spectroscopy. Thus, $\nu_{\mathrm{OH}}^{\mathrm{H}}$ of the H-bound structure can appear in the ADIR spectrum. The isolation of these two contributions in the current experimental approach is the key to clarify the correct ion core dynamics of $\mathrm{PhOH}-\mathrm{Ar}$.
Although all observations upon excitation and ionization of $\mathrm{PhOH}-\mathrm{Ar}$ are now qualitatively understood and explained, several important questions remain to be addressed in future work. In particular, realistic and accurate potential energy surfaces of $\mathrm{PhOH}-\mathrm{Ar}$ have to be determined for the $\mathrm{S}_{1}$ and $\mathrm{D}_{0}$ states, in order to calculate reliable Franck-Condon factors responsible for the individual transitions between the two electronic states. This will answer the question of which states of the H-bound structure can directly be ionized from the $\pi$-bound $S_{1}$ origin level. Moreover, it will give a modified assignment of the intermolecular transitions observed in the ZEKE/MATI spectra, in particular for transitions above the $\pi \rightarrow \mathrm{H}$ isomerization barrier. The solution of the vibrational Schrödinger equation for the three-dimensional intermolecular potential will provide a reliable estimate of the intermolecular frequencies of the triple minimum potential in Fig. 5, and in particular the energy difference between $\operatorname{IE}_{0}(\pi)$ and the barrier. The assignment of the intermolecular transitions observed in the ZEKE and MATI spectra is strongly connected with the understanding of the reaction path. The reaction path should be expressed by a linear combination using all the three intermolecular coordinates. Along the path, the potential must be flat above the reaction barrier, and only the remaining two coordinates can be bound. The open potential does not provide discrete and well separated vibrational levels as those detected in the ZEKE and MATI spectra. The assignment of ZEKE and MATI spectra using three intermolecular modes, therefore, has to be reconsidered, although in this paper we have followed the previously given assignment derived assuming a strongly bound $\pi$-bound structure to label peaks in the MATI spectrum. Experimentally, the time response of the $\nu_{\mathrm{OH}}^{\mathrm{H}}$ band can confirm the direct ionization of the $\mathrm{H}$-bound structure. When $\mathrm{PhOH}-\mathrm{Ar}$ is ionized $\sim 20 \mathrm{~cm}^{-1}$ below $\operatorname{IE}_{0}(\pi), \nu_{\mathrm{OH}}^{\mathrm{H}}$ must appear simultaneously with the ionization if the direct ionization of the $\mathrm{H}$-bound structure is realized. Moreover, the PIE spectrum should be measured further down to possibly detect the $\mathrm{IE}_{0}(\mathrm{H})$ level, which is required for the experimental determination of the depth of the H-bound well.

\section{Summary}

We developed a new laser spectroscopic technique, MATI-IR spectroscopy, which enables us to selectively measure the IR spectra of a molecular ion core in high- $n$ Rydberg states of clusters, and studied the mechanism of the $\pi \rightarrow \mathrm{H}$ site switching reaction in the $\mathrm{PhOH}^{+}-\mathrm{Ar}$ cluster, a benchmark system for the investigation of intermolecular interactions. This technique allowed us to unambiguously resolve the fundamental inconsistency between the ZEKE spectra and the picosecond timeresolved IR spectra of the phenol-Ar cation reported previously. The MATI-IR spectrum measured at the $\operatorname{IE}_{0}(\pi)$ level shows only the $\nu_{\mathrm{OH}}^{\pi}$ band, while those at intermolecular modes give both the $\nu_{\mathrm{OH}}^{\mathrm{H}}$ and the $\nu_{\mathrm{OH}}^{\pi}$ transitions. From these observations, it is concluded that the $\pi \rightarrow \mathrm{H}$ site switching reaction occurs only from vibrationally excited levels and has a very small activation 
barrier from the zero-point level of the $\pi$-bound local minimum. This barrier height is lower than $14 \mathrm{~cm}^{-1}$, which is the lowest-energy vibrationally excited level of the $\pi$-bound cation. The REMPI-IR spectra recorded simultaneously with the MATIIR spectra, on the other hand, always provide the $\nu_{\mathrm{OH}}^{\mathrm{H}}$ band even for ionization below the $\operatorname{IE}_{0}(\pi)$ level. This observation is rationalized by direct ionization of the $\mathrm{H}$-bound structure from the $\pi$-bound $\mathrm{S}_{1}$ state via adiabatic $\pi \rightarrow \mathrm{H}$ site switching. All the observed results are consistently interpreted including this previously ignored mechanism of the direct ionization from the $\pi$-bound neutral to the $\mathrm{H}$-bound structure in the cation, which has not been expected because of the large geometrical change. These results demonstrate that the ionization processes affect the final structures of the ionic system. A thorough re-assignment of the intermolecular vibrations in the ZEKE spectra, high level calculations for the potential energy surfaces of $S_{1}$ and $D_{0}$, and time-resolved IR spectroscopy including the time-response of $\nu_{\mathrm{OH}}^{\mathrm{H}}$ are required and now in progress.

\section{Acknowledgements}

This work was supported in part by the Core-to-Core Program 22003 from the Japan Society for the Promotion of Science (JSPS), KAKENHI on Innovative Area (2503) "Studying the Function of Soft Molecular Systems by the Concerted Use of Theory and Experiment", the Cooperative Research Program of "Network Joint Research Center for Materials and Devices", from the Ministry of Education, Culture, Sports, Science and Technology (MEXT), Japan, and Deutsche Forschungsgemeinschaft (DFG, DO 729/4-2). F.M. was supported by the Fonds National de la Recherche, Luxembourg (AFR/1357660). We acknowledge initial experimental support by Takaki Sato, Takashi Nakamura, Yuri Sakata, Katsuya Kikuchi and Shinya Tanaka.

\section{References}

1 N. Gonohe, H. Abe, N. Mikami and M. Ito, J. Phys. Chem., 1985, 89, 3642-3648.

2 M. Mons, J. L. Calvé, F. Piuzzi and I. Dimicoli, J. Chem. Phys., 1990, 92, 2155-2165.

3 A. Fujii, T. Sawamura, S. Tanabe, T. Ebata and N. Mikami, Chem. Phys. Lett., 1994, 225, 104-107.

4 I. Kalkman, C. Brand, T.-B. C. Vu, W. L. Meerts, Y. N. Svartsov, O. Dopfer, K. Müller-Dethlefs, S. Grimme and M. Schmitt, J. Chem. Phys., 2009, 130, 224303.

5 N. Solcà and O. Dopfer, Chem. Phys. Lett., 2000, 325, 354-359.

6 N. Solcà and O. Dopfer, J. Phys. Chem. A, 2001, 105, 5637-5645.

7 N. Solcà and O. Dopfer, Eur. Phys. J. D, 2002, 20, 469-480.

8 M. Fujii and O. Dopfer, Int. Rev. Phys. Chem., 2012, 31, 131-173.

9 A. Takeda, H.-S. Andrei, M. Miyazaki, S. Ishiuchi, M. Sakai, M. Fujii and O. Dopfer, Chem. Phys. Lett., 2007, 443, 227-231.
10 O. Dopfer, Z. Phys. Chem., 2005, 219, 125-168.

11 S. Ishiuchi, M. Miyazaki, M. Sakai, M. Fujii, M. Schmies and O. Dopfer, Phys. Chem. Chem. Phys., 2011, 13, 2409-2416.

12 N. Solcà and O. Dopfer, Chem. Phys. Lett., 2003, 369, 68-74.

13 A. Patzer, J. Langer, H. Knorke, H. Neitsch, O. Dopfer, M. Miyazaki, K. Hattori, A. Takeda, S. Ishiuchi and M. Fujii, Chem. Phys. Lett., 2009, 474, 7-12.

14 J. Černý, X. Tong, P. Hobza and K. Müller-Dethlefs, Phys. Chem. Chem. Phys., 2008, 10, 2780-2784.

15 J. Černý, X. Tong, P. Hobza and K. Müller-Dethlefs, J. Chem. Phys., 2008, 128, 114319.

16 N. Solcà and O. Dopfer, J. Mol. Struct., 2001, 563/564, 241-244.

17 M. Schmies, A. Patzer, M. Fujii and O. Dopfer, Phys. Chem. Chem. Phys., 2011, 13, 13926-13941.

18 X. Zhang and J. L. Knee, Faraday Discuss., 1994, 97, 299-313. 19 C. E. H. Dessent, S. R. Haines and K. Müller-Dethlefs, Chem. Phys. Lett., 1999, 315, 103-108.

20 C. E. H. Dessent and K. Müller-Dethlefs, Chem. Rev., 2000, 100, 3999-4022.

21 S. R. Haines, C. E. H. Desent and K. Müller-Dethlefs, J. Electron Spectrosc. Relat. Phenom., 2000, 108, 1-11.

22 S. Ullrich, G. Tarczay and K. Müller-Dethlefs, J. Phys. Chem. A, 2002, 106, 1496-1503.

23 M. Miyazaki, S. Tanaka, S. Ishiuchi, O. Dopfer and M. Fujii, Chem. Phys. Lett., 2011, 513, 208-211.

24 M. Miyazaki, A. Takeda, S. Ishiuchi, M. Sakai, O. Dopfer and M. Fujii, Phys. Chem. Chem. Phys., 2011, 13, 2744-2747.

25 S. Ishiuchi, M. Sakai, Y. Tsuchida, A. Takeda, Y. Kawashima, O. Dopfer, K. Müller-Dethlefs and M. Fujii, J. Chem. Phys., 2007, 127, 114307.

26 W. Habenicht, G. Reiser and K. Müller-Dethlefs, J. Chem. Phys., 1991, 95, 4809-4820.

27 A. Armentano, M. Riese, M. Taherkahani, M. B. Yezzar, K. Müller-Dethlefs, M. Fujii and O. Dopfer, J. Phys. Chem. A, 2010, 114, 11139-11143.

28 X. Tong, A. Armentano, M. Riese, M. B. Yezzar, S. M. Pimblott, K. Müller-Dethlefs, S. Ishiuchi, M. Sakai, A. Takeda, M. Fujii and O. Dopfer, J. Chem. Phys., 2010, 133, 154308.

29 A. Fujii, A. Iwasaki, T. Ebata and N. Mikami, J. Phys. Chem. A, 1997, 101, 5963-5965.

30 E. Fujimaki, A. Fujii, T. Ebata and N. Mikami, J. Chem. Phys., 1999, 110, 4238-4247.

31 O. Dopfer, G. Reiser, K. Müller-Dethlefs, E. W. Schlag and S. D. Colson, J. Chem. Phys., 1994, 101, 974-989.

32 M. Miyazaki, A. Takeda, M. Schmies, M. Sakai, K. Misawa, S. Ishiuchi, F. Michels, K. Müller-Dethlefs, O. Dopfer and M. Fujii, Phys. Chem. Chem. Phys., 2014, 16, 110-116.

33 H. J. Neusser and H. Krause, Chem. Rev., 1994, 94, 1829-1843.

34 N. Solcà and O. Dopfer, J. Phys. Chem. A, 2001, 105, 5637-5645.

35 R. J. Lipert and S. D. Colson, J. Chem. Phys., 1990, 92, 3240-3241. 
36 A. J. Gotch, A. W. Garrett, D. L. Severance and T. S. Zwier, Chem. Phys. Lett., 1991, 178, 121-129.

37 H. Tachikawa and M. Igarashi, J. Phys. Chem. A, 1998, 102, 8648-8656.

38 A. Courty, M. Mons, I. Dimicoli, F. Piuzzi, M.-P. Gaigeot, V. Brenner, P. de Pujo and P. Millié, J. Phys. Chem. A, 1998, 102, 6590-6600.
39 N. Solcà and O. Dopfer, Chem. Phys. Lett., 2001, 347, 59-64.

40 M. Miyazaki, A. Fujii, T. Ebata and N. Mikami, Chem. Phys. Lett., 2001, 349, 431-436.

41 J. N. Bardsley, Chem. Phys. Lett., 1967, 1, 229-232.

42 J. Berkowitz, Photoabsorption, Photoionization and Photoelectron Spectroscopy, Academic Press, New York, 1979. 